\title{
Understanding seizure dogs
}

David C. Spencer, MD

In their article "Pseudoseizure Dogs," Dr. Krauss and coworkers (Neurology 2007:68:308-309) look at the use of service dogs to assist patients with epilepsy and other related conditions. The authors found that several of their patients with seizure response dogs did not suffer from epileptic seizures, but in fact had "pseudoseizures" (now more commonly called "nonepileptic seizures").

Nonepileptic seizures can be difficult to distinguish from epileptic seizures: they may look exactly like epileptic seizures. The difference is that during a nonepileptic seizure, there are no abnormal electrical discharges in the brain of the type seen in epileptic seizures. Nonepileptic seizures can have many causes; however, the most common cause is a psychiatric illness. In these illnesses (the most common called a "conversion disorder"), psychological stresses come out as physical symptoms. Usually, the nonepileptic seizures are not under the person's control.

\section{What are seizure alert and seizure response dogs?}

Seizure response dogs are animals that respond to a person having a seizure. Usually, the dog will be helpful in some way during the seizure itself. The dog may respond by instinct, or it can be trained for this purpose. Such animals may be able to alert others that a seizure is happening or will stay with the person during a seizure to help avoid injury. Finally, as the person is awakening from the seizure, the presence of the seizure dog can be both reassuring and reorienting to the patient. Many patients report feeling more confident, knowing that they have some type of assistance for their seizures.

A seizure alert dog is one that can alert the patient or family member before a seizure begins, or in the very early phase of a seizure, so that appropriate steps may be taken to make sure that the patient is safe. We do not understand how dogs might be able to do this, and we do not know much about how good they are at predicting seizures correctly. Mostly we know that people with seizure alert dogs report feeling "better" and "more confident" and enjoy the companionship of their seizure dog.

\section{How was the study done?}

The authors reported the stories of six patients at their center who had seizure dogs, and they told the stories of four of these people in detail because of the interesting findings.

\section{What did the study find?}

After careful study, the authors found that four of the patients with seizure response dogs did not have epileptic seizures, but had "pseudoseizures" or "nonepileptic seizures" (NES). In each of these people, they felt that there was a psychological cause for the seizure-like events rather than an abnormal electrical discharge in the brain. These patients needed different treatments to help control their events than people with epilepsy.

Of these four people with NES, three patients recovered from their NES and one moved away (so the authors do not know what happened to her). One person returned the dog to its training program, and the other three kept the dogs as pets.
The authors also reported limited information about two patients with epilepsy whose dogs stay with them when a seizure happens. These patients found that the dog's responses were helpful. They also noted the benefits of companionship with the dog to help them deal with a medical problem that has no easy cure and lasts for a long time.

\section{What does this mean for people with seizures?}

Seizure response dogs are helpful for some people with epilepsy, as reported in this and other studies. This is an area that deserves more study so that we can understand better how some dogs might be able to tell when a seizure is coming and see how good they are at doing this. Dogs may also be good companions for people with chronic disease. Because highly trained dogs are expensive, it makes good sense to understand what they can and cannot do and where they could be best used. In this way, we may be able to assign seizure dogs to the people who would benefit most.

It is important to recognize that some patients with seizure response dogs may not have epileptic seizures, but may be suffering from a different condition (NES) that causes behavior similar to seizures, but is caused by a mental health problem. The authors suggest that doctors should first clarify the underlying diagnosis. They feel that many people might benefit from the companionship and emotional support of a pet, but that highly trained service animals should closely match the patient's condition. 


\section{What is a seizure?}

A seizure is a disruption in the normal electrical activity of the brain. Normally the brain is very active, passing electrical messages back and forth between nerve cells. When a person has a seizure, there is abnormal firing of nerve cells and the messages become jumbled in part or all of the brain.

A seizure may cause a variety of different symptoms, such as twitching or shaking in an arm, leg, one side of the face, or the whole body; repetitive movements or gestures; confusion; feelings of fear or other emotions; hallucinations (odd smells, tastes, sounds, or seeing things that are not there); loss ofconsciousness; and convulsions. [Adapted from Patient Page: Szabo CA. Risk of fetal death and malformation related to seizure medications. Neurology 2006;67:E6-E7.]

\section{What is epilepsy?}

Epilepsy is an episodic recurrence of seizures that are not due to fever, active infection, drug effects, or other triggering causes. It may be caused by a variety of conditions that injure a part or all of the brain, such as problems in development of the brain that occurs before birth, inherited disorders of the brain or nervous system, brain trauma, brain tumors, stroke, infections, and poisoning. About $70 \%$ of cases have no known cause.

Each year there are 125,000 new cases of epilepsy. About 2.5million people in the United States have some form of epilepsy. [Adapted from Patient Page: Szabo CA. Risk of fetal death and malformation related to seizure medications. Neurology 2006;67:E6-E7.]

\section{What are nonepileptic} seizures ("pseudoseizures")? Nonepileptic seizures are temporary disturbances of neurologic function. Although they may look like epileptic seizures, there are several differences. The most important difference is that the brain wave activity recorded on an EEG test is normal during a nonepileptic seizure. In other words, the event is NOT caused by an abnormal electrical discharge in the brain.

There are two types of nonepileptic seizures: physiologic and psychogenic. Physiologic nonepileptic seizures are less common. In these events, there is a clear physical cause of the seizure event, but it is not epileptic and there are no abnormal electrical discharges in the brain. There are several causes of physiologic nonepileptic seizures, including fainting (also called syncope), TIAs (small strokes), sleep disorders, and migraines. For instance, it is common for an individual to experience jerking movements of the arms or legs during a fainting episode. This can sometimes be mistaken for a seizure, but has a different cause and different treatment. Psychogenic nonepileptic seizures are much more common, and these may be connected to many psychiatric illnesses. Usually it is felt that these events are a way that psychological stress comes out as physical symptoms. People with this condition may appear to lose consciousness and may have shaking or other activity that looks very much like an epileptic seizure, but again, there is no abnormal electrical activity in the brain. Most often the patient with this condition is not aware of the connection to psychological stresses and does not have control over the episodes.

The treatment of nonepileptic seizures first depends on the correctly identifying the cause of the symptoms. Then the treatment can focus on the specific physical or mental health issue causing the episodes. This may involve counseling or "talk therapy," or use of medications to treat depression or anxiety that often go along with psychogenic nonepileptic seizures. Our usual epilepsy medications do not work in people with nonepileptic seizures. If a person with a diagnosis of epilepsy does not respond to antiseizure medications, the physician should always consider the possibility of nonepileptic seizures.

\section{For more information}

For more information about seizures, epilepsy, and seizure response dogs, please contact the Epilepsy Foundation of America: http://www.epilepsyfoundation.org 


\section{Neurology}

\section{Understanding seizure dogs}

David C. Spencer

Neurology 2007;68;E2-E3

DOI 10.1212/01.wnl.0000255054.50937.13

This information is current as of January 22, 2007

\section{Updated Information \&}

Services

Permissions \& Licensing

Reprints including high resolution figures, can be found at:

http://n.neurology.org/content/68/4/E2.full

Information about reproducing this article in parts (figures,tables) or in its entirety can be found online at:

http://www.neurology.org/about/about_the_journal\#permissions

Information about ordering reprints can be found online:

http://n.neurology.org/subscribers/advertise

Neurology ${ }^{\circledR}$ is the official journal of the American Academy of Neurology. Published continuously since 1951, it is now a weekly with 48 issues per year. Copyright . All rights reserved. Print ISSN: 0028-3878. Online ISSN: 1526-632X.

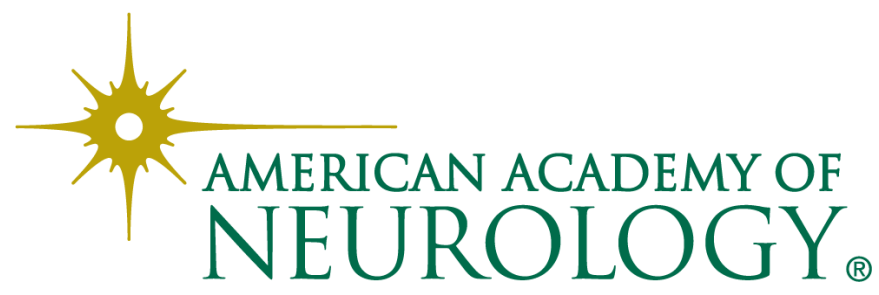

